\title{
Scaling-based prediction of magnetic anisotropy in grain-oriented steels
}

\author{
MARIUSZ NAJGEBAUER \\ Faculty of Electrical Engineering \\ Czestochowa University of Technology \\ e-mail:mariusz.najgebauer@gmail.com
}

(Received: 28.02.2017, revised: 27.04.2017)

\begin{abstract}
The paper presents the scaling-based approach to analysis and prediction of magnetic anisotropy in grain-oriented steels. Results of the anisotropy scaling indicate the existence of two universality classes. The hybrid approach to prediction of magnetic anisotropy, combining the scaling analysis with the ODFs method, is proposed. This approach is examined in prediction of angular dependencies of magnetic induction as well as magnetization curves for the 111-35S5 steel. It is shown that it is possible to predict anisotropy of magnetic properties based on measurements in three arbitrary directions for $\phi=0^{\circ}, 60^{\circ}$ and $90^{\circ}$. The relatively small errors between predicted and measured values of magnetic induction are obtained.
\end{abstract}

Key words: grain-oriented steels, magnetic anisotropy, ODFs method, scaling analysis

\section{Introduction}

Electrical steels are still the basic magnetic materials used as magnetic cores in electrical machines such as motors, generators and transformers. There are two groups of electrical steels: non-oriented and grain-oriented ones. Typical non-oriented steels are $0.35-0.8 \mathrm{~mm}$ thick with 0-3\% silicon content, while grain-oriented steels are $0.23-0.35 \mathrm{~mm}$ with $2.9-3.2 \%$ silicon content. Non-oriented steels are usually considered as isotropic materials, with the same magnetic properties in any direction. Grain-oriented steels exhibit strong anisotropy of magnetic properties, created by many rolling and re-crystallization production steps [1-7]. In fact, non-oriented steels may reveal a significant level of anisotropy $[2,8]$, which can affect the efficiency of electrical devices. For this reason, the anisotropy of magnetic properties is apart from peak induction and power loss - one of the most important parameters determining material usefulness for magnetic circuits of electrical machines.

There are many models describing an anisotropy phenomenon in magnetic materials. However, the models used in practical computations should allow one to predict magnetic anisotropy based on input data (e.g. measurements) obtained for selected directions, which reduces troublesome sample preparations and measurements for intermediate directions. This require- 
ment is met by the phenomenological models based on the coenergy concept as well as the theory of Orientation Distribution Functions (ODFs).

The coenergy-based model of magnetic anisotropy have been proposed by Péra [9] and developed by Biró [10] and Chwastek [11,12]. This description is given by the ellipse-like equation:

$$
\left(\frac{\boldsymbol{B} \cdot \boldsymbol{e}_{1}}{B_{1}(H)}\right)^{n}+\left(\frac{\boldsymbol{B} \cdot \boldsymbol{e}_{2}}{B_{2}(H)}\right)^{n}=1,
$$

where: $\boldsymbol{B}$ is a magnetic induction vector, $\boldsymbol{e}_{1,2}$ are unit vectors in the rolling and the transverse direction, $B_{1,2}(H)$ describes magnetization curve in these directions and $n$ is a model exponent.

The original elliptical model (for $n=2$ ) does not provide satisfactory results for the direction of the worst magnetic properties. In the case of $n \approx 1.4$, Eq. (1) takes the form more appropriate for the description of magnetic anisotropy in all directions [10-12].

The coenergy-based model allows one to predict magnetization curves for any direction using measurements carried out for the rolling and the transverse direction, which are often provided by steel manufacturers. However, this method may cause some problems in numerical implementations.

Another approach to the anisotropy modeling is based on the theory of Orientation Distribution Functions (ODFs), which allows one to correlate magnetic properties and the crystallographic texture of the sample. This approach has been developed by Bunge $[13,14]$ and advanced by de Campos $[15,16]$. Recently, Chwastek examined this method for grain-oriented electrical steels $[17,18]$. In general, the ODFs method allows one to describe the anisotropy of magnetic properties (such as magnetization, coercivity or power loss) using the three first ODFs coefficients

$$
A=A_{0}+A_{1} \cos 2 \phi+A_{2} \cos 4 \phi,
$$

where: $A_{0,1,2}$ are the ODFs coefficients given by the following expressions:

$$
\begin{aligned}
& A_{0}=0.25 \cdot\left[A\left(0^{\circ}\right)+A\left(90^{\circ}\right)+2 A\left(45^{\circ}\right)\right], \\
& A_{1}=0.5 \cdot\left[A\left(0^{\circ}\right)-A\left(90^{\circ}\right)\right], \\
& A_{0}=0.25 \cdot\left[A\left(0^{\circ}\right)+A\left(90^{\circ}\right)-2 A\left(45^{\circ}\right)\right] .
\end{aligned}
$$

It results from (2) and (3), that measurements of the magnetic property carried out in the three arbitrary directions $\left(\phi=0^{\circ}, 45^{\circ}\right.$ and $\left.90^{\circ}\right)$ are sufficient to predict values of this property for intermediate directions. It should be noted that the value of the ODFs coefficients vary depending on the excitation [17]. Thus, even though the ODFs method provides satisfactory results of the magnetic anisotropy prediction, it requires measurements in the three directions carried out for each level of the excitation.

The paper presents a hybrid approach to prediction of magnetic anisotropy, combining the scaling analysis with the ODFs method. The proposed approach should result in further simplification of anisotropy prediction due to using universal values of the ODFs coefficients. This approach will be examined for the grain-oriented steel. 


\section{Samples and measurements}

Samples for measurements were prepared from the grain-oriented steel grade 111-35S5, produced by Stalprodukt S.A., Bochnia. The samples had a shape of stripes $(305 \pm 0.5 \mathrm{~mm}$ long, $30 \pm 0.2 \mathrm{~mm}$ wide and $0.35 \mathrm{~mm}$ thick) and were cut from the steel sheet at a different angle to the rolling direction, i.e. for $\phi=0^{\circ}, 15^{\circ}, 30^{\circ}, 45^{\circ}, 60^{\circ}, 75^{\circ}$ and $90^{\circ}$. Measurements were carried out using the $25 \mathrm{~cm}$ Epstein frame according to the international standard IEC 60404-2:1996-03, Part 2: Methods of measurement of the magnetic properties of electrical steel sheet and strip by means of an Epstein frame. Angular dependencies of magnetic induction for different level of magnetic field strength are depicted in Fig. 1. The measurements indicate a strong anisotropy of magnetic properties of the examined steel. For $H>200 \mathrm{~A} / \mathrm{m}$, the worst magnetic properties are observed for the angle close to $\phi=55^{\circ}$, what is determined by the Goss texture of grain-oriented steels [5-7, 12, 17, 19-21]. It should be noted that the angular dependence of magnetic induction for $H=100 \mathrm{~A} / \mathrm{m}$ has a different trajectory compared to the other dependencies - the worst direction of magnetic properties is for $\phi=90^{\circ}$. Similar results were reported in [21-25]. This effect may result from the change of the magnetization mechanism for magnetic field strength of about $200 \mathrm{~A} / \mathrm{m}$. For $H<200 \mathrm{~A} / \mathrm{m}$ the domain-wall translation is responsible for the magnetization process, while in the case of $H>200 \mathrm{~A} / \mathrm{m}$ the rotation of magnetization vectors is the main magnetization mechanism [17].

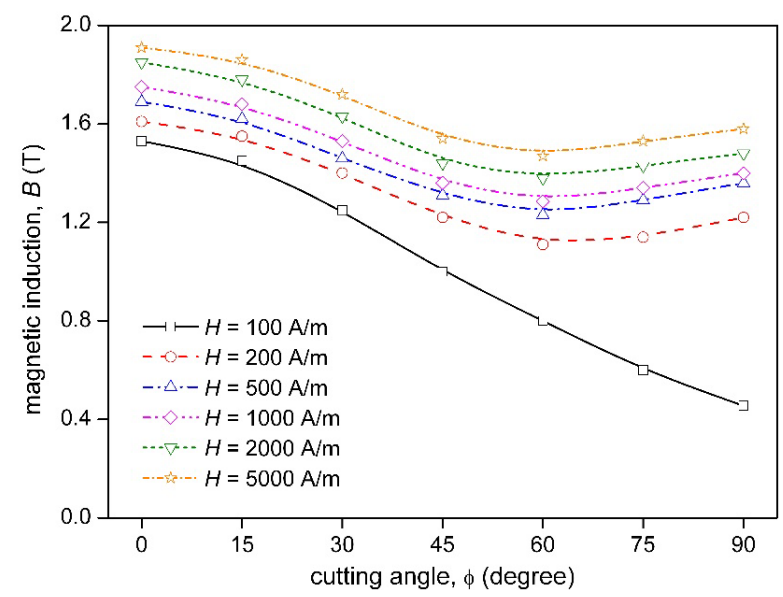

Fig. 1. Magnetic induction versus the cutting angle for the 111-35S5 steel

\section{Scaling analysis of magnetic anisotropy}

In modern physics, the scaling theory is applied usually in the analysis of critical behavior and phase transitions [26-29]. The scaling analysis is also used in materials science, including the study of magnetic material properties such as power loss [30-34], coercivity [35-37], magnetic viscosity [38-40] or hysteresis loops [41-45]. 
Referring to the scaling theory as well as the measurements (see Fig. 1), it is proposed to describe the anisotropy of magnetic properties by a functional relationship of a cutting angle $\phi$ and magnetic field strength $H$

$$
B=f(\phi, H) .
$$

The relationship (4) is assumed to be a generalized homogenous function, which is defined as:

$$
\lambda^{x} B=f\left(\lambda^{y} \phi, \lambda^{z} H\right),
$$

where: $\lambda$ is a scaling coefficient and $x, y, z$ are any numbers $[28,29]$. For $\lambda=H^{-1 / z}$, the relationship (5) is transformed as follows:

$$
\begin{aligned}
H^{-x / z} B & =f\left(H^{-x / z} \phi, 1\right), \\
\frac{B}{H^{\psi}} & =F\left(\frac{\phi}{H^{\varphi}}\right),
\end{aligned}
$$

and finally the scaled form of (4) is obtained

$$
B_{H}=F\left(\phi_{H}\right),
$$

where: $B_{H}$ and $\phi_{H}$ are magnetic induction and a cutting angle scaled with respect to $H, \psi$ and $\varphi$ are scaling exponents and $F\left(\phi_{H}\right)=f\left(\phi_{H}, 1\right)$ denotes a so-called scaling function. In the previous studies, a scaling function was usually represented by Maclaurin series [30-37].

In the considered study, the scaling function $F\left(\phi_{H}\right)$ is supposed to be represented by the following series:

$$
B_{H}=B_{0}+B_{1} \cos 2 \phi_{H}+B_{2} \cos 4 \phi_{H}+B_{3} \cos 6 \phi_{H}+\ldots,
$$

which results from the angular dependence of the magnetic properties anisotropy. Referring to the ODFs methods (2), only the three first coefficients of (9) may be taken into account. Thus, in further computations the reduced expression describing scaled magnetic induction will be examined

$$
B_{H}=B_{0}+B_{1} \cos 2 \phi_{H}+B_{2} \cos 4 \phi_{H} .
$$

\section{Results and discussion}

\subsection{Scaling of magnetic anisotropy}

The measured dependencies of magnetic induction $B$ versus the cutting angle $\phi$ were scaled in respect to magnetic field strength $H$ according to (10). The scaling coefficients $\psi, \varphi$ and $B_{0-2}$ were estimated using the least square method in such a way that allowed collapsing of 
measurement data onto a single dependency. Only in the case of data collapse, the coefficient values may be considered as universal. The scaling of magnetic induction measurements for the examined study is presented in Fig. 2. It can be observed that data points for $H=100 \mathrm{~A} / \mathrm{m}$ are not collapsed and diverge significantly from the data collapse curve. It suggests that the measurements at low magnetic field strength (about $100 \mathrm{~A} / \mathrm{m}$ ) belong to one universality class, while the other measurements - to another universality class. The existence of different universality classes were reported previously in analysis of power loss in La-containing alloys [33] and minor hysteresis loops in Finmet type cores [45].

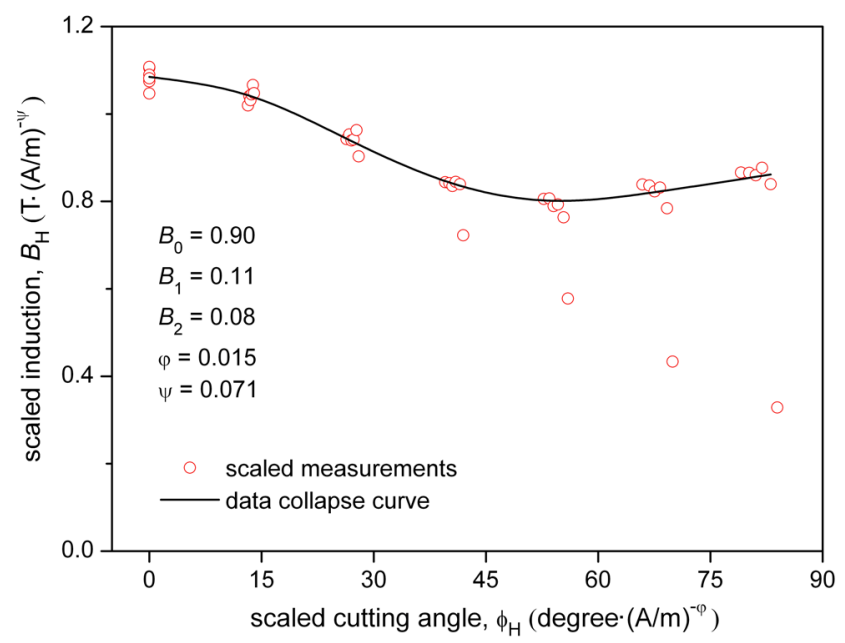

Fig. 2. Scaling and data collapse of the magnetic anisotropy for the 111-35S5 steel

Referring to the scaling theory, each class of universality should be characterized by the specific set of coefficients. Thus, values of the coefficients $\psi, \varphi$ and $B_{0-2}$ can be considered as universal in the measurement range $H=200 \div 5000 \mathrm{~A} / \mathrm{m}$. Concluding, the presented results confirm the validity of scaling procedures in the analysis of magnetic anisotropy.

\subsection{Prediction of magnetic anisotropy}

The proposed scaling should allow one to prediction the anisotropy of magnetic properties, including such levels of magnetic field strength $H$ or cutting angles $\phi$, which haven't been used in estimation of the scaling coefficients. This assumption was verified in two case studies, concerning the prediction of:

1) Angular dependencies of magnetic induction at various levels of magnetic field strength,

2) Magnetization curves at various cutting angles.

In the first case study, scaling coefficients were estimated from the angular dependencies of magnetic induction, measured for all cutting angles and selected levels of magnetic field strength, i.e. $H_{\min }=200 \mathrm{~A} / \mathrm{m}$ and $H_{\max }=5000 \mathrm{~A} / \mathrm{m}$. The angular dependencies of magnetic induction were then predicted for intermediate levels of magnetic field strength using (10), transformed to the following form: 


$$
B=H^{\psi} \cdot\left(B_{0}+B_{1} \cos 2 \frac{\phi}{H^{\varphi}}+B_{2} \cos 4 \frac{\phi}{H^{\varphi}}\right)
$$

The prediction results for $H=500,1000$ and $2000 \mathrm{~A} / \mathrm{m}$ are depicted in Fig. 3. The relative errors between the measured and predicted values of magnetic induction did not exceed $5 \%$. The error values are presented in Table 1.

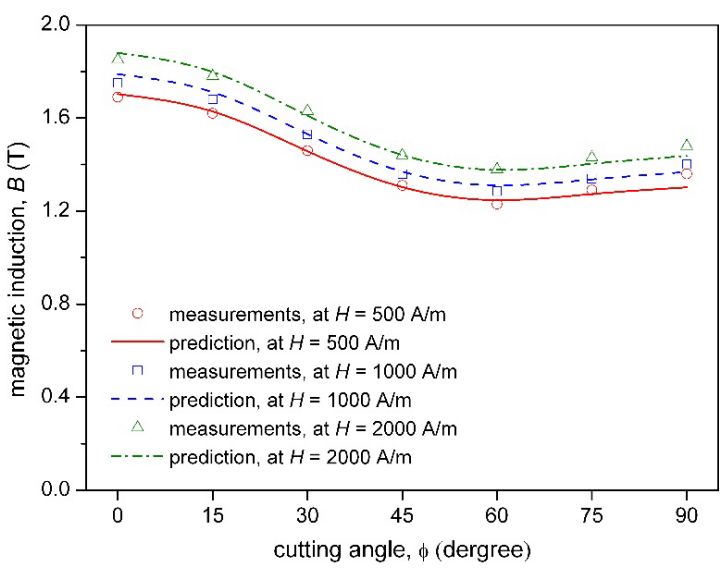

Fig. 3. Prediction of magnetic induction versus the cutting angle for the 111-35S5 steel

Table 1. Prediction errors for the case study 1

\begin{tabular}{l|c|c|c|c|c|c|c}
\hline Cutting angle $\phi$ (degree) & $\mathbf{0}$ & $\mathbf{1 5}$ & $\mathbf{3 0}$ & $\mathbf{4 5}$ & $\mathbf{6 0}$ & $\mathbf{7 5}$ & $\mathbf{9 0}$ \\
\hline Errors (\%) at $\boldsymbol{H}=\mathbf{5 0 0} \mathbf{A} / \mathbf{m}$ & 0.77 & 0.49 & 0.24 & 0.50 & 1.41 & 1.35 & 4.26 \\
\hline Errors (\%) at $\boldsymbol{H}=\mathbf{1 0 0 0} \mathbf{A} / \mathbf{m}$ & 2.25 & 1.83 & 0.09 & 0.77 & 1.99 & 0.27 & 2.29 \\
\hline Errors (\%) at $\boldsymbol{H}=\mathbf{2 0 0 0} \mathbf{A} / \mathbf{m}$ & 1.61 & 1.00 & 1.23 & 0.06 & 0.22 & 1.86 & 2.91 \\
\hline
\end{tabular}

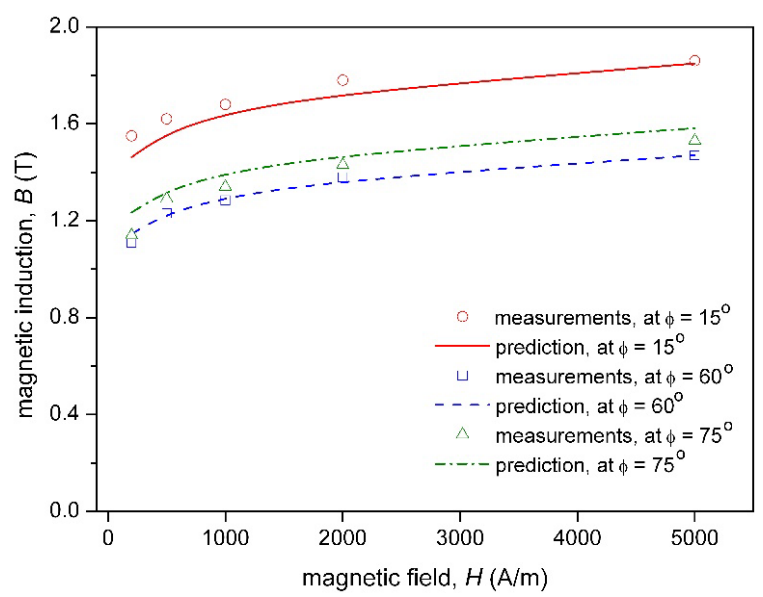

Fig. 4. Prediction of magnetization curves for the 111-35S5 steel 
In the second case study, the scaling coefficients were estimated according to the ODFs method, i.e. from measurements for cutting angles $\phi=0^{\circ}, 60^{\circ}$ and $90^{\circ}$. The cutting angle $\phi=60^{\circ}$ was chosen instead of $\phi=45^{\circ}$ (the ODFs method), because it represented the direction of worst magnetic properties. The magnetization curves, predicted for chosen cutting angles, are depicted in Fig. 4, while corresponding relative errors are presented in Table 2. The prediction errors in this case study did not exceed $6 \%$.

Table 2. Prediction errors for the case study 2

\begin{tabular}{l|c|c|c|c|c}
\hline Magnetic field $\boldsymbol{H}(\mathbf{A} / \mathbf{m})$ & $\mathbf{2 0 0}$ & $\mathbf{5 0 0}$ & $\mathbf{1 0 0 0}$ & $\mathbf{2 0 0 0}$ & $\mathbf{5 0 0 0}$ \\
\hline Errors (\%) at $\phi=\mathbf{1 5}^{\circ}$ & 5.70 & 3.53 & 2.15 & 2.86 & 0.61 \\
\hline Errors (\%) at $\phi=\mathbf{6 0}^{\circ}$ & 3.24 & 0.05 & 0.99 & 0.78 & 0.01 \\
\hline Errors (\%) at $\phi=\mathbf{7 5}^{\circ}$ & 2.79 & 1.48 & 0.59 & 0.01 & 0.03 \\
\hline
\end{tabular}

Values of the scaling coefficients, estimated from different measurement ranges, are presented in Table 3. These are comparable in all considered studies except the scaling exponent $\varphi$, whose value depends on the measurement range. This issue will be the subject of further research.

Table 3. Estimated values of scaling coefficients

\begin{tabular}{l|c|c|c|c|c}
\hline Measurements & $\boldsymbol{B}_{\mathbf{0}}$ & $\boldsymbol{B}_{\mathbf{1}}$ & $\boldsymbol{B}_{\mathbf{2}}$ & $\boldsymbol{\varphi}$ & $\boldsymbol{\Psi}$ \\
\hline Full range & 0.90 & 0.11 & 0.08 & 0.015 & 0.071 \\
\hline Reduced range (case study 1) & 0.90 & 0.13 & 0.07 & 0.004 & 0.071 \\
\hline Reduced range (case study 2) & 0.86 & 0.10 & 0.09 & -0.010 & 0.074 \\
\hline
\end{tabular}

The results presented in this section confirm that the scaling-based method allows one to predict the magnetic anisotropy in a wide range of magnetic field strength or cutting angles, with relatively low errors. Moreover, the proposed method generates universal values of the coefficients $B_{0-2}$, whereas in the case of the ODFs method - the coefficients $A_{0-2}$ should be estimated separately for each level of magnetic field strength.

\section{Conclusions}

In the paper, the use of scaling analysis in description of magnetic anisotropy in electrical steels has been proposed. This approach was examined for the grain-oriented steel 111-35S5. The angular dependencies of magnetic induction for $H=200 \div 5000 \mathrm{~A} / \mathrm{m}$ were collapsed onto a single curve, while a significant divergence of measurements for $H=100 \mathrm{~A} / \mathrm{m}$ was observed. For this reason, the existence of two universality classes was postulated. Estimated values of the scaling coefficients could be considered as universal only in the limited range of magnetic field strength $H=200 \div 5000 \mathrm{~A} / \mathrm{m}$. Nevertheless, the presented results confirmed the validity of scaling procedures in the analysis of magnetic anisotropy. 
The hybrid method for magnetic anisotropy prediction, combining the scaling analysis with the ODFs method, was proposed. This approach was verified in two case studies, concerning prediction of angular dependencies of magnetic induction as well as magnetization curves for various cutting angles. It was shown that it is possible to predict anisotropy of magnetic properties based on measurements for three arbitrary angles $\phi=0^{\circ}, 90^{\circ}$ and $60^{\circ}$, corresponding to rolling, transverse and worst magnetic properties direction, respectively. The relatively small errors of the scaling-based prediction of magnetic anisotropy were obtained. The proposed method generates coefficients, which have universal values for a wide range of magnetic field strength. In contrast, the coefficients in the ODFs method should be estimated separately for each level of magnetic field strength. For this reason, the scaling-based prediction of magnetic anisotropy should be considered as a simpler and more universal method, compared to the ODFs one.

In future research, the proposed method will be examined in the analysis of magnetic anisotropy in the range of low magnetic field strength $(\mathrm{H}<200 \mathrm{~A} / \mathrm{m})$ in order to investigate its scaling behavior.

\section{Acknowledgements}

The author would like to thank Professor Jan Szczygłowski for providing measurements of angular magnetic properties as well as a useful, inspiring and motivating discussion. The author is also grateful to Professor Krzysztof Chwastek for his comments pertaining to magnetic anisotropy.

\section{References}

[1] Fiorillo F., Anisotropy and magnetization process in soft magnets: Principles, experiments, applications, Journal of Magnetism and Magnetic Materials, vol. 304, pp. 139-144 (2006).

[2] Tumański S., Investigations of the anisotropic behaviour of SiFe steel, Journal of Magnetism and Magnetic Materials, vol. 254-255, pp. 50-53 (2003).

[3] Soiński M., Bak Z., Bargiel P., Anisotropy of magnetic properties in thin electrical sheets, IEEE Transactions on Magnetics, vol. 26, no. 6, pp. 3076-3079 (1990).

[4] Soiński M., Moses A.J., Anisotropy of iron-based soft magnetic materials (Chapter 4), Handbook of magnetic materials, vol. 8, North-Holland Elsevier, pp. 325-414 (1995).

[5] Fryskowski B., Experimental evaluation of magnetic anisotropy in electrical steel sheets, Journal of Magnetism and Magnetic Materials, vol. 320, pp. 515-552 (2008).

[6] Emura M., de Campos M.F., Landgraf F.J.G., Teixeira J.C., Angular dependence of magnetic properties of 2\% silicon electrical steel, Journal of Magnetism and Magnetic Materials, vols. 226-230, pp. 1524-1526 (2001).

[7] Mazgaj W., Warzecha A., Influence of electrical steel sheet textures on their magnetization curves, Archives of Electrical Engineering, vol. 62, no. 3, pp. 425-437 (2013).

[8] Najgebauer M., Szczygłowski J., Kapłon A., Soft magnetic materials for energy-efficient electric motors, the $12^{\text {th }}$ Conference on Selected Problems of Electrical Engineering and Electronics (WZEE2015), Kielce, Poland, pp. 1-4 (2015).

[9] Péra T., Ossart F., Waeckerlé Th., Numerical representation for anisotropic materials based on coenergy modeling, Journal of Applied Physics, vol. 73, no. 10, pp. 6784-6786 (1993).

[10] Biró O., Ausserhofer S., Preis K., Chen Y., A modified elliptic model of anisotropy in nonlinear magnetic materials, Proceedings of $8^{\text {th }}$ International Symposium on Electric and Magnetic Field (EMF’2009), Mondovi, Italy, pp. 25-26 (2009). 
[11] Chwastek K., Najgebauer M., Szczygłowski J., Wilczyński W., Modelling the influence of anisotropy on magnetic properties in grain-oriented steels, Przegląd Elektrotechniczny, vol. 87, no. 3, pp. 126-128 (2011).

[12] Chwastek K., Anisotropic properties of non-oriented steel sheets, IET Electronic Power Applications, vol. 7, no. 7, pp. 575-579 (2013).

[13] Bunge H.J., Texture analysis in materials science: mathematical methods, Butterworth (1982).

[14] Bunge H.J., The basic concepts of texture investigation in polycrystalline materials, Steel Research, vol. 62, no. 12, pp. 530-541 (1991).

[15] de Campos M.F., Anisotropy of steel sheets and consequence for Epstein test, Proceeding of XVIII IMEKO World Congress "Metrology for a Sustainable Development", Rio de Janeiro, Brazil, p. 6 (2006).

[16] Yonamine T., de Campos M.F., Castro N.A., Landgraf F.J.G., Modeling magnetic polarization $J_{50}$ by different methods, Journal of Magnetism and Magnetic Materials, vol. 304, pp. e589-e592 (2006).

[17] Chwastek K., Baghel A., De Campos M., Kulkarni S.V., Szczygłowski J., A Description for the Anisotropy of Magnetic Properties of Grain-oriented Steels, IEEE Transactions on Magnetics, vol. 51, no. 12, pp. 6000905.1-5 (2015).

[18] Chwastek K., Baghel A.P.S., Wodzyński A., Kulkarni S.V., Anisotropic properties of electrical steels, $6^{\text {th }}$ International Conference on Computational Problems of Electrical Engineering (CPEE), Lviv, Ukraine, pp. 21-23 (2015).

[19] Cornut B., Kedous-Lebouc A., Waeckerlé Th., From metallurgy to modelling electrical steels: a multiple approach to their behaviour and used based on physics and experimental investigations, Journal of Magnetism and Magnetic Materials, vol. 160, pp. 102-108 (1996).

[20] Pluta W., Angular properties of specific total loss components under axial magnetization in grainoriented electrical steel, IEEE Transactions on Magnetics, vol. 52, issue 4, no. 6300912 (2016).

[21] Paltanea G., Paltanea V., Gavrila H., Popovici D., Ionescu G., Magnetic anisotropy in grain oriented steels cut through mechanical punching and electro-erosion technologies, AIP Conference Proceedings, vol. 1809, pp. 020041.1-6 (2017).

[22] Fiorillo F., Dupré L.R., Appino C., Rietto A.M., Comprehensive model of magnetization curve, hysteresis loops, and losses in any direction in grain-oriented $\mathrm{Fe}-\mathrm{Si}$, IEEE Transactions on Magnetics, vol. 38, no. 3, 1467-1475 (2002).

[23] Fiorillo F., Appino C., Beatrice C., Garsia F., Magnetization process under generically directed field in $\mathrm{GO} \mathrm{Fe}-(3 \mathrm{wt} \%)$ Si laminations, Journal of Magnetism and Magnetic Materials, vols. 242-245, pp. 257-260 (2002).

[24] Tumański S., Handbook of magnetic measurements, CRC Press (2011).

[25] Tumański S., Investigations of two-dimensional properties of selected electrical steel samples by means of the Epstein method, $7^{\text {th }}$ Workshop on 2D Measurements, Lüdenscheid, Germany, pp. 151-157 (2002).

[26] Widom B., Equation of state in the neighbourhood of the critical point, Journal of Chemical Physics, vol. 43, pp. 3898-3905 (1965).

[27] Stanley H.E. Introduction to phase transitions and critical phenomena, Clarendon Press (1971).

[28] Klamut J., Durczewski K., Sznajd J., Introduction to the physics of phase transition, Zakład Narodowy im. Ossolińskich (in Polish), Wrocław (1978).

[29] Stanley H.E., Scaling, universality and renormalization: three pillars of modern critical phenomena, Reviews of Modern Physics, vol. 71, no. 2, pp. S358-S366 (1999).

[30] Sokalski K., Szczygłowski J., Najgebauer M., Wilczyński W., Losses scaling in soft magnetic materials, COMPEL: The International Journal for Computation and Mathematics in Electrical and Electronic Engineering, vol. 26, no. 3, pp. 640-649 (2007).

[31] Najgebauer M., Scaling theory and its chosen applications in electromagnetism, Przegląd Elektrotechiczny, vol. 84, no. 12, pp. 213-216 (2008).

[32] Najgebauer M., Application of fractional scaling in modelling of magnetic power losses, Acta Physica Polonica A, vol. 128, pp. 107-110 (2015). 
[33] Gozdur R., Najgebauer M., Measurements and scaling analysis of power losses in La-containing alloys, Journal of Electrical Engineering, vol. 66, no. 7/s, pp. 37-40 (2015).

[34] Najgebauer M., Scaling-based analysis and modelling of power losses in amorphous and nanocrystalline alloys, Acta Physica Polonica A, vol. 131, no. 5, pp. 1125-1127 (2017).

[35] Najgebauer M., The concept of scaling analysis in describing the properties of soft magnets, Solid State Phenomena, vol. 220-221, pp. 646-651 (2015).

[36] Najgebauer M., Sokalski K., Szczygłowski J., The modified scaling procedure in coercivity modelling, Archives of Electrical Engineering, vol. 64, no. 3, pp. 351-359 (2015).

[37] Najgebauer M., Fractional scaling of magnetic coercivity in electrical steels, Acta Physica Polonica A, vol. 131, no. 4, pp. 633-635 (2017).

[38] Mayergoyz I.D., Adly A.A., Korman C., Huang M., Krafft C., Scaling and data collapse in magnetic viscosity, Journal of Applied Physics, vol. 85, pp. 4358-4360 (1999).

[39] Mayergoyz I.D., Adly A.A., Huang M.W., Krafft C., Scaling and data collapse in magnetic viscosity (creep) of superconductors, IEEE Transactions on Magnetics, vol. 36, no. 5, pp. 3208-3210 (2000).

[40] Adedoyin A., Andrei P., Data collapse and viscosity in three-dimensional magnetic hysteresis modeling, IEEE Transactions on Magnetics, vol. 44, no. 11, pp. 3165-3168 (2008)

[41] Takahashi S., Kobayashi S., Shishido T., A scaling power-law relation in magnetic minor hysteresis loops in Fe and Ni metals, Journal of Physics: Condensed Matter, vol. 20, pp. 035217/ $1-035217 / 6(2008)$.

[42] Kobayashi S., Kikuchi N., Takahashi S., Kikuchi H., Kamada Y., Scaling analysis of minor hysteresis loops in ferromagnets with large uniaxial anisotropy, Journal of Magnetism and Magnetic Materials, vol. 322, no. 9-12, pp. 1515-1518 (2010).

[43] Kobayashi S., Tsukidate S., Kamada Y., Kikuchi H., Ohtani T., Investigation of scaling laws in frequency-dependent minor hysteresis loops for ferromagnetic steels, Journal of Magnetism and Magnetic Materials, vol. 324, no. 2, pp. 215-221 (2012).

[44] Kobayashi S., Takahashi S., Ishibashi Y., Hysteresis scaling behavior in a remanent magnetization state, IEEE Transactions on Magnetics, vol. 48, no. 4, pp. 3165-3168 (2012).

[45] Varga L.K., Kováč J., Minor loop scaling rules for Finemet type soft magnetic cores, Acta Physica Polonica A, vol. 126, pp. 156-157 (2014). 